\title{
¿EXISTE PROTECCIÓN REAL A LOS DERECHOS HUMANOS DEL ADULTO MAYOR? EL DISCURSO LEGAL
}

\section{IS A REAL PROTECTION OF HUMAN RIGHTS OF THE ELDERLY? THE LEGAL DISCOURSE}

\author{
Marlon Mora Jiménez
}

\begin{abstract}
RESUMEN
En este artículo se desarrolla una explicación del discurso del especialista en leyes $y$ sus operadores sin dejar de lado su manual de operaciones básico en este caso: la ley. De este modo, se hace un pasaje por la situación de la vejez en el ámbito jurídico internacional y nacional donde se analiza la Ley 7935.
\end{abstract}

PALABRAS CLAVE: COSTA RICA * VEJEZ * DERECHOS HUMANOS * PROTECCIÓN LEGAL * CONDICIONES DE VIDA

\section{ABSTRACT}

This article develops an explanation of the law specialist speech and its operators without neglecting its core operations manual in this case: the law. By this way, it raises the situation of elder people in the international legal framework and national where it discusses the 7935 Act.

KEYWORDS: COSTA RICA * OLD AGE * HUMAN RIGHTS * LEGAL PROTECTION * LIVING CONDITIONS

Profesor de la Universidad Nacional y la Universidad de Costa Rica. Consultor de Naciones Unidas, ILANUD.

marlon78@hotmail.com 


\section{INTRODUCCIÓN}

\section{UNA LEGISLACIÓN DE PROEZAS SIN CONSECUENCIAS...}

\section{(DISCURSO DEL LEGISLADOR)}

-ABOGADOS Y OPERADORES DE JUSTICIA-

El artículo que se presenta a continuación obedece a un proyecto de investigación que desarrollo en la Universidad Nacional sobre las Representaciones sociales de la vejez. Además cuenta con algunos insumos propios de una investigación doctoral ${ }^{1}$ para así presentarles cual es el discurso que utilizan los legisladores y operadores de la justicia en el marco de la vejez. Al finalizar se comentan algunas conclusiones y reflexiones propias de esta experiencia.

Para hablar de la potencia de un discurso del legislador habría que escudriñar el contexto en los que desde nuestra América se han planteado los derechos humanos de las personas adultas mayores y específicamente en Costa Rica en materia legal con leyes dirigidas a ese grupo poblacional.

Años atrás un decálogo olvidado en Suramérica pregonó una ancianidad con esperanza. El 28 de agosto de 1948, Eva Perón proclamó en la Argentina los Derechos de la Ancianidad, con el anhelo de que fueran incluidos en las leyes fundamentales que regulaban la vida nacional. Así, fueron incorporados a la Constitución de 1949, y poco después, en la Asamblea Especial de Naciones Unidas, habiendo sido tomados para sí por otras naciones.

El decálogo, ese olvidado en la historia, incluye: derecho a la asistencia (familiar o estatal en el caso de los desamparados); derecho a la vivienda; derecho a la alimentación; derecho al vestido; derecho a la salud física; derecho al esparcimiento; derecho al trabajo; derecho a la expansión y derecho al respeto.

Ante esto es grato descubrir que poco después de proclamada la Declaración Universal

1 "La construcción de la imagen de la persona adulta mayor en Costa Rica a través del discurso en los medios de comunicación colectiva” — graduación de honor Cum Laude y recomendación para publicación íntegra del trabajo de investigación en Universidad Pablo de Olavide, Sevilla, España-. de los Derechos Humanos, que cumplirá 62 años en el 2010, ya existía en Argentina una preocupación por la protección del adulto mayor, punto a rescatar desde el garantismo ${ }^{2}$ de los derechos humanos proclamados.

Para que algo similar sucediese hubo que esperar al año 1965 cuando en Estados Unidos, para atender las diversas necesidades del anciano, se editó el Acta del Adulto Mayor (The Older American Act) que autorizó el establecimiento de la Administración de Envejecimiento.

Otro de los antecedentes en pro de la vejez se encontró en la Comunidad Europea cuando la Asociación Internacional de Ciudadanos Ancianos y la Federación Europea para Personas Ancianas dictaron mediante sus representantes el primer enunciado que se conoce sobre derechos del adulto mayor, en 1961, en Washington, Estados Unidos. Esta iniciativa destaca la existencia física y cultural, la vida económica y social y la autonomía (Proño, 1991). Pero, el enunciado no llegó a más y careció de apoyo en el ámbito internacional.

Más tarde, serían las Naciones Unidas con el Plan de Acción Internacional de Viena sobre el Envejecimiento - que cuenta con la ratificación de 154 países incluido Costa Rica- realizado en 1982 . En esa propuesta de acción se trató la situación del adulto mayor ${ }^{3}$. De ahí, se concluyó la necesidad de iniciar un Programa Internacional de Acción encaminado a garantizar la seguridad social y la economía de las personas de edad, así como de ofrecérseles oportunidades para que contribuyan en el

El garantismo es una corriente jurídica que parte del reconocimiento de los derechos fundamentales de los individuos $y$ de su efectiva protección y tutela como la piedra de toque del diseño constitucional del Estado.

El crecimiento que experimenta la población
mayor de 60 años es un desafío, así como la
convicción de que la Declaración Universal de
Derechos Humanos se aplique íntegramente a las
personas de este grupo vulnerable. Además del
reconocimiento de la importancia de la calidad
de vida y de la longevidad y la importancia de que
las personas mayores puedan permanecer en el
seno de sus propias familias, disfrutar de una vida
plena, saludable, segura y satisfactoria, como inte-
grantes de la sociedad (ONU, 1982).


desarrollo de la sociedad. Lamentablemente, los acuerdos no fueron ratificados por la totalidad de las naciones participantes por lo que sus alcances son mínimos.

Por otro lado, sería importante mencionar que la Comisión de Desarrollo Social de las Naciones Unidas se reunió en 1991 para discutir la aprobación de una Declaración de los Derechos Humanos de las Personas de Edad Avanzada. Entre los argumentos utilizados para fundamentar la necesidad de la aprobación se encontraron:

$\diamond$ Que la declaración cambiaría el rumbo hacia un mundo en el que el valor de las personas no disminuiría con los años.

$\diamond$ La necesidad urgente de atender los problemas económicos, sociales y políticos que generan los cambios de la estructura de la población.

$\diamond$ Dotar a las personas de edad de instrumentos específicos que les permitan defenderse de los actos que violentan sus derechos.

$\diamond$ Brindar un trato preferencial a esta población como se le ha brindado al niño, a la mujer, el indígena o la persona con discapacidad.

Sin embargo, una vez más, esta iniciativa por el adulto mayor fracasó por la oposición de algunos países, entre ellos Alemania. De esta forma, lograron sustituir con una serie de principios en cuya redacción quedó diluida la esencia de los derechos. Y como siempre en derechos humanos, la historia quedó reducida en un "deberían ser"; perdiéndose así la fuerza de exigibilidad del derecho (Nunberg, 1991).

En materia legal, la situación de las personas adultas mayores ha sido relegada a declaraciones y convenciones de carácter general. A estas alturas no encontramos un instrumento jurídico internacional que se encargue de tutelar derechos específicos a favor de esta población a no ser el Artículo 17 del Protocolo Adicional a la Convención Americana en materia de Derechos Económicos, Sociales y Culturales, Protocolo de San Salvador, el cual dice:

\section{Artículo 17. Protección de los Ancianos}

Toda persona tiene derechos a protección especialmente durante su ancianidad. En tal cometido, los Estados partes se comprometen a adoptar de manera progresiva las medidas necesarias a fin de llevar estos derechos a la práctica $y$ en particular a:

Proporcionar instalaciones adecuadas, así como alimentación y atención médica especializada a las personas de edad avanzada que carezcan de ella y no se encuentren en condiciones de proporcionársela por sí mismas.

Ejecutar programas laborales específicos, destinados a conceder a los ancianos la posibilidad de realizar una actividad adecuada a sus capacidades respetando su vocación o deseos.

Estimular la formación de organizaciones sociales destinadas a mejorar la calidad de vida de los ancianos.

Es conocida y ya bastamente criticada la separación entre derechos civiles y políticos con respecto a los económicos, sociales y culturales, distanciados y aislados en dos protocolos facultativos hijos de la guerra fría. Este maniqueísmo que viene de 1966, año de creación de ambos instrumentos internacionales, llevó a incorporar el elemento de progresividad ${ }^{4}$ en el Pacto de San Salvador como medida reticente para el cumplimiento de esos derechos, para los cuales el Estado parte debe asegurar un ejercicio directo y sistemático para su cumplimiento. En este caso en particular, la "devastadora" progresividad no se hizo esperar.

4 La progresividad en el derecho se va a tener como prestaciones del Estado que deberá asumir en la "medida de sus posibilidades" dejando a la deriva derechos "fundamentales" para la concreción de un proyecto de vida a criterio único y unilateral del Estado $y$ sus funciones.

Se plantea como devastadora debido a que socava la posibilidad de llevar a cabo un proyecto de vida digna. La relativa acción de los Estados quedará a 
Sin embargo, en algunos países sí se han realizado esfuerzos para que se brinde protección especial a las personas adultas mayores por la vía de la legislación específica. Así encontramos en Guatemala, Ecuador y México leyes para ese grupo etario.

En Honduras, en el año 2000, se aprobó la Ley de la Tercera Edad que permite a las personas mayores de 60 años recibir descuentos del 25 por ciento en el pago de servicios públicos, medicinas y el ingreso a centros de diversión y entretenimiento. Sin embargo, la falta de sanciones impide que ese beneficio se cumpla ${ }^{6}$.

Por doquier surge la necesidad de una normativa que cumpla con una "verdadera" protección a las necesidades específicas de las personas adultas mayores. Es un hecho que el principio de simplificación no puede ser tomado como referencia a la hora de establecer una ley específica, es necesario tomar conciencia de la determinación etnosociocéntrica que, de partida, hipoteca toda concepción de la sociedad, de la cultura, del hombre (Morin, 1984).

En Costa Rica, como parte de este proceso, se reconoció la inexistencia de una legislación que contemplará los derechos del adulto mayor. Anteriormente, se utilizaron sólo decretos, los cuales con algunas excepciones, son débiles comparados con la existencia de una ley específica.

Así, se aprobó la Ley de Protección Integral al Anciano, enviada a la Asamblea Legislativa en abril de 1992. Empero, tuvo que esperar siete años para que fuese Ley de la República - 1999 - llamada Ley Integral del Adulto Mayor 7935.

propio criterio, lo cual no permite exigir de forma directa un derecho económico o un derecho social si el Estado, con anterioridad, plantea que las medidas a tomar siguen un orden progresivo, sin prisa.

Tiempos del Mundo (Periódico). "Presente a margo, futuro incierto”. Honduras. (14/02/2002).

\section{EL LEGISLADOR Y SU MANUAL DE OPERACIONES}

En el informe de labores de la Defensoría de los Habitantes de la República de Costa Rica, correspondiente al período 1999-2000, se reconoció que en materia de protección jurídica de la población adulta mayor, la más significativa conquista que experimentó el país, a propósito de las acciones realizadas en el marco de la celebración del Año Internacional de las Personas Adultas Mayores (1999), se encontró en la promulgación de la Ley Integral para la Persona Adulta Mayor Ley 7935 y otras reformas legales en materia de transporte remunerado de personas, vivienda y recursos económicos, en procura del mejoramiento de la calidad de vida de este segmento poblacional de conformidad con el respeto de sus derechos fundamentales. Por esto, sería importante retomar algunas definiciones que da la Ley Integral del Adulto Mayor (1999) para saber hasta dónde la aplicación de la ley es real.

El artículo dos de esta ley contiene la definición de lo que se concibe como persona adulta mayor, la cual destaca una importante consideración en relación con nuestro país, ya que es usual que se defina al adulto mayor como aquella persona que sobrepasa los sesenta años de edad. En esta ley, por el contrario, se le define a partir de los sesenta y cinco años, con lo cual se reconocen indicadores demográficos $y$ de salud importantes que destacan al país. De acuerdo con la Ley Integral del Adulto Mayor 7935, de 1999, adulto mayor se considera a toda persona de sesenta y cinco años o más. (Artículo $2^{\circ}$, capítulo II).

Entendido desde una lógica biológica sostenida con los niveles de productividad de un país, se pone de manifiesto cuál debe ser la edad para designar desde el lenguaje del legislador y cómo sería políticamente correcto llamarle en este caso a los de más edad: persona adulta mayor.

Se sobreentiende que el triángulo poblacional impide que la pensión sea a una edad más temprana por los costos productivos y la baja en el Producto Interno Bruto. Sin embargo, esta podría ser una sospecha relacionada 
con el artículo tres: capítulo uno ${ }^{7}$, inciso $\mathrm{i}^{8}$; artículo cuatro ${ }^{9}$ punto $\mathrm{b}^{10}$; artículo $11^{11}$ (los cuales hablan sobre los niveles de producción y consumo).

Si se va a estos artículos específicamente, se puede entender bien la necesidad de que los mayores participen en el proceso productivo del país, por las capacidades de consumo que pueden tener $y$ la necesidad de que estos roles sean asumidos, para que desde sus espacios entren en la participación de ese modelo de economía que no destacaría el círculo hacia la vejez - porque lo mejor de llegar a viejo sería contar con dinero para ser parte de ese proceso productivo-.

Por otro lado, una perspectiva muy diferente se encuentra en una economía de la solidaridad (Hinkelammert, 2008) donde esa vuelta hacia los valores plantea una forma de entender los recursos con una visión solidaria tratando de recoger las diferentes experiencias de hacer economía - producción, distribución, consumo $y$ ahorro- que se caracterizan por vertebrarse en torno al apoyo ${ }^{12}$.

Desde esta óptica serían emprendimientos solidarios tanto aquellos de carácter asociativo (una cooperativa, una asociación, una comunidad), como aquellos que se organizan no para beneficio propio, sino orientados por el bien común o el bien de algún

7

Artículo 3.- Derechos para mejorar la calidad de vida.

i) La participación en el proceso productivo del país, de acuerdo con sus posibilidades, capacidades, condición, vocación y deseos.

Artículo 4.- Derechos laborales.

b) Contar con los horarios laborales y los planes vacacionales adecuados a sus necesidades, siempre que tal adecuación no perjudique la buena marcha de la entidad empleadora.

Artículo 11.- Beneficios.

Ese apoyo entendido como la solidaridad (del latín solidum) a su vez debe entenderse en un doble sentido: primero como todo aquello que hacemos en conjunto con otros, dando lugar a la asociatividad $y$, segundo como todo comportamiento que tenga en cuenta el bienestar de un tercero. sector desfavorecido de la sociedad que harían de ese proceso productivo uno inclusivo del círculo de la vida — niño, adulto y mayor-.

\section{SI PRODUCEN: ¡MEJOR!}

Lamentablemente, los beneficios en la propia Ley 7935 se ven desde una definición incompleta de ocio donde se destaca la capacidad para consumir. Tratando de explicarlo desde una fórmula, se podría decir que ocio, más consumo, más dinero, dan como resultado acceso a la salud en el caso de ese modelo productivo; una persona saludable con planes de ocio consume más (ocio + consumo $+\$=$ salud), dejando la solidaridad a un lado, por ende, el círculo de la vida saca del juego a quienes no tienen: plata (dinero).

Se legisla, asimismo, acerca de los centros que tienen a cargo o están relacionados con la temática de la persona adulta mayor, tales como la acreditación, que consiste en el reconocimiento formal de la competencia de una institución, organización o persona física para realizar tareas o tipos de tareas específicas. También la ley define lo que se concibe como hogar privado sustituto: establecimiento privado donde habitan personas adultas mayores, financiadas o no con fondos públicos. $\mathrm{Su}$ administración está a cargo de organizaciones no gubernamentales, como asociaciones calificadas de bienestar social.

\section{HOTELES DE ESTRELLAS}

Estas han sido creadas como nuevas casas de estancia para planearles el retiro obligado a las personas adultas mayores. A esas modalidades creadas para sacarlos de su propia casa se les llaman hogares de ancianos o bien asilos, donde de manera pública o privada se les ofrece la posibilidad de una vivienda y de un alimento donde puedan pasar sus últimos días.

Sin embargo, estas casas de cuido no son la mejor opción para una persona adulta mayor acostumbrada a la tierra, a su propia casa o bien a poder hacer lo que "quería" sin tener que pedir permiso en un lugar donde nada es suyo. 
Ciertamente, para algunos mayores este nuevo lugar se convierte en peculiaridad y sus nuevas compañías ya no serán allegados de la familia sino extraños con los cuales les tocará convivir mañana, tarde $y$ noche. Pero también es destacable la oportunidad que les brindan a muchos adultos mayores espacios como estos, que se convierten en toda una experiencia de vida.

En algunos casos, claro está, el lugar protagónico que ocupaban en sus casas cambia hacia un escenario donde son abandonados $y$ excluidos del sistema, aunque en el discurso oficial se alimente la creación de estos espacios bajo la premisa de la tranquilidad, quietud, seguridad $y$ salubridad que ofrecen.

En el caso del sistema público, efectivamente estos hogares de ancianos han venido a funcionar para recuperar físicamente a muchos adultos mayores; pero, quedan relegados muchos otros derechos como la independencia $y$ la disponibilidad. Otra historia representan los asilos privados que gozan de buena infraestructura y capacidad económica para ofrecer a sus inquilinos - adultos mayores - un hotel de cinco estrellas.

Pero en ambos casos, el abandono es una de las experiencias que viven los adultos mayores; donde su casa ya no es más suya y no les queda más que aferrarse a un cuarto en una habitación cualquiera. Abandonados, olvidados, en soledad, a expensas de nuevas compañías y sin nada en la bolsa, en algunos casos las personas adultas mayores son excluidas del contrato social, donde ya no producen ni son sinónimo de riqueza.

Otros aspectos que aborda la Ley 7935 son los de atención integral, ayudas técnicas para personas con discapacidad, servicios de atención general o especializada, institucionalizada, interna o ambulatoria a domicilio, de rehabilitación física, mental o social y de asistencia, en general, para las personas adultas mayores.

Se abordan importantes concepciones como la de riesgo social, situación de mayor vulnerabilidad en que se encuentran las personas adultas mayores cuando presentan factores de riesgo que, de no ser tratados, les producen daños en la salud, así como la de seguridad social, entendida como el conjunto de prestaciones sanitarias, sociales y económicas que contribuyen a dotar a las personas de una vida digna $y$ plena.

En lo que se refiere a la violencia contra las personas adultas mayores, se define como cualquier acción $\mathrm{u}$ omisión, directa o indirecta, ejercida contra ellas, que produzca, como consecuencia, el menoscabo de su integridad física, sexual, psicológica o patrimonial. Este podría ser uno de los apartados más importantes porque según datos del Consejo Nacional de la Persona Adulta Mayor (CONAPAM) ${ }^{13}$ sus bienes patrimoniales son de los más violentados en las personas adultas mayores.

Sin dejar de lado el aumento de un 20 por ciento en agresiones, en el año 2000, los casos atendidos en el Hospital Geriátrico Raúl Blanco Cervantes aumentaron un 20,5 por ciento en relación con 1999, cuando se registraron 117 pacientes ingresados por violencia.

Esta es la violencia directa que reciben por falta de una cultura de protección real de derechos humanos que les proteja, a pesar de la existente Ley Integral del Adulto Mayor (1999), porque los medios internos de protección de tales derechos, difícilmente permiten a un anciano hacer todo el papeleo que implica una acusación en los tribunales de justicia.

En el Hospital, el Comité de Estudio Integral del Anciano Abandonado y Agredido detectó, en el año 2000, una mayor frecuencia en abusos de tipo psicológico (92 casos), físico (30 casos) y patrimonial (30 casos). Mientras tanto, la Ley 7935 establece que protege al adulto mayor en esos ámbitos delegando esa tarea en el Estado y algunas otras instituciones. Para ejemplo solamente se podrían citar los artículos 3 y 6 , respectivamente.

Según el artículo tres: Derechos para mejorar la calidad de vida. Toda persona adulta mayor tendrá derecho a una mejor calidad de vida, mediante la creación y ejecución de programas que promuevan:

En comunicado de prensa se informó que apenas en abril del 2009, el CONAPAM ya tenía 1500 denuncias de abuso contra personas adultas mayores. 
a) El acceso a la educación, en cualquiera de sus niveles, $y$ a la preparación adecuada para la jubilación.

\section{EDUCACIÓN DE MENTIRAS...}

Cómo garantizarles acceso a la educación si la falta de estos programas en el nivel formal es pan de cada día. La academia no ha desarrollado planes de estudio dirigidos, especialmente, para esa población; todo lo contrario, los pone en desventaja al ubicarlos en una clase donde la diferencia de años con sus compañeros los hace claudicar y dejar los estudios. Si es difícil retomar los estudios para un joven de 19 años en un colegio o escuela, mucho más difícil para una persona de más de 65 años. Si vamos a darles acceso a la educación, debemos hacerlo fácil $o$ al menos accesible.

En otros ámbitos como el universitario (Universidad Nacional —UNA- o Universidad de Costa Rica - UCR-) se pueden identificar programas interesantes, pero siempre con una perspectiva desde el apartamiento o el alejamiento.

Para académicos universitarios prepararse para la etapa jubilatoria no es para nada motivante, en especial después de pasar a la categoría de pensionado porque según las políticas universitarias (Universidad Nacional, Universidad de Costa Rica e Instituto Tecnológico de Costa Rica -ITCR-), es conveniente estimular nuevos cuadros y no contratar a profesores jubilados, que valga destacar muchos de ellos o ellas aún no son catalogados dentro del rubro de personas adultas mayores a falta de la edad para cumplir ese requisito -65 años-.

\section{VIEJOS: ¿SUJETOS DE CRÉDITO?}

Otro de los puntos que podría causar mayor interés desde el ámbito de acceso a igualdad de oportunidades es el punto: d) El acceso al crédito que otorgan las entidades financieras públicas y privadas.

Está claro que si una persona adulta mayor solicita una tarjeta de crédito el acceso es limitadísimo, en especial si ya cuenta con una pensión porque como bien, esta ya no es embargable. De esta manera, aunque son reglas que no están escritas en los bancos, la política subyacente es no abrir el crédito a este grupo poblacional.

Para Isabel Hidalgo Ramírez, alajuelense de 67 años, la obtención de una tarjeta de crédito fue un asunto complicado; después de hacer solicitudes en varios bancos estatales el único que le dio esa posibilidad fue Banca Promérica, uno de los bancos privados de nuestro país ${ }^{14}$.

Sobre la línea del acceso a una mejor calidad de vida:

e) El acceso a un hogar sustituto $u$ otras alternativas de atención, con el fin de que se vele por sus derechos e intereses, si se encuentra en riesgo social.

En la actualidad, nuestro país cuenta con un total de 10 por ciento de personas adultas mayores, de las cuales un 2 por ciento se encuentra institucionalizado. Si no se puede enfrentar ese porcentaje, ¿cómo se hará en los próximos años con el crecimiento de esa población?

f) La atención hospitalaria inmediata, de emergencia, preventiva, clínica y de rehabilitación.

Sobre la atención hospitalaria en general el sistema estatal no ofrece inmediatez, asunto destacable en la prevención de enfermedades $y$ tratamiento de males propios de la edad.

g) La pensión concedida oportunamente, que le ayude a satisfacer sus necesidades fundamentales, haya contribuido o no a un régimen de pensiones.

Efectivamente, si una persona solicita una pensión aunque no haya contribuido con el régimen después de realizar un agotador trámite puede lograr obtener su derecho - de 30 mil colones, menos de 55 dólares-. Pero,

Para hacerlo en cualquier Banco Estatal necesitaría de al menos tres fiadores con salarios mensuales superiores al suyo. 
este monto no será lo suficiente para completar sus necesidades básicas; de allí que se encuentre a muchas personas adultas mayores en trabajos informales como cuido de carros, ventas de frutas y otros que no dignifican esa etapa de la vida.

Del mismo modo, en el artículo 6 se hace referencia a la integridad:

Derecho a la integridad. Las personas adultas mayores tendrán derecho a que se respete su integridad física, psíquica y moral. Este derecho comprende la protección de su imagen, autonomía, pensamiento, dignidad $y$ valores.

Todo está escrito y es Ley de la República, pero en la práctica según datos del Comité de Estudio Integral del Anciano Abandonado y Agredido (CEINAA), las agresiones aumentan. La ley dice que se le respetará su integridad física, psicológica hasta patrimonial y en la práctica, el CEINAA se llena de casos y las calles de las cabeceras de provincia nos abofetean en las noches, cuando cerca de basureros, arropados con cartones o bolsas de basura, duermen decenas de ancianos. ¿Dónde están sus derechos, si es que acaso les queda alguno? ¿Dónde están las instituciones que la Ley indica? ¿Dónde está el apoyo real del Estado a esas instituciones y personas?

\section{CONCLUSIONES}

En resumen, la legislación existe pero no es garantía de respeto de derechos para los adultos mayores. El problema que hay no es la ley sino la poca capacidad de acción que se les da a los individuos mayores para exigir sus derechos y la falta de conocimiento de la mayoría de sus conciudadanos de tales derechos.

Cabe también señalar que se deriva de esta ley una preocupación proteccionista para ese grupo poblacional, donde se regulan aspectos de salud, de cuido, pero no el del rescate de la persona íntegra que aún puede participar de la vida cultural y social cotidiana, de alternativas para cuando ya se superan los sesenta años y aún pesan sobre los adultos mayores responsabilidades de otros o de su propia sobrevivencia.
Pese a tan valioso instrumento jurídico, lastimosamente muchas de las premisas expresadas en la ley en la realidad son letra muerta por la gran cantidad de violaciones de derechos humanos que se ven día a día para este grupo en situación de vulnerabilidad. Una muestra de ello: el aumento en el año 2000 de agresiones contra adultos mayores (con un incremento de 20 por ciento).

Algunos de estos casos se refieren a la situación de personas que se encuentran subsistiendo en condiciones precarias e indignas para cualquier ser humano. Tal es el caso de un albergue ubicado en la capital, San José, el cual fue denunciado tres veces — 1998, 1999, 2000sin que se haya contado con el adecuado abordaje técnico de los órganos competentes.

Una vez más, se aprecia cómo en derechos humanos, la ley no lo es todo y mucho menos suficiente para la realidad social y económica de países como el nuestro. Lo anterior puede documentarse con informaciones de noticias que señalan el grave sufrimiento que atraviesan los adultos mayores ${ }^{15}$.

Otro punto importante es destacar que a la luz de la creación de la ley, instituciones encargadas por velar por la promoción y la protección del adulto mayor han hecho conciencia sobre su importancia en la sociedad.

Posteriormente con la promulgación de la Ley 7935, no se han visto cambios radicales en el trabajo por las personas mayores. Más bien, nuestro país ha sufrido con casos como el sucedido el 18 de julio del 2000 en el Hogar de Ancianos de Tilarán, donde murieron quemadas 17 personas adultas mayores. De esa forma, quedó en evidencia que en el país no existen medidas preventivas para el cuido de adultos mayores.

Fernando Morales Martínez ${ }^{16}$, médico y director del Hospital Geriátrico Raúl Blanco

Un dato que justifica lo anterior lo vivió en carne propia una anciana colombiana, paciente terminal de Sida, que esperó desnuda la muerte al frente del hospital siendo ignorada por la sociedad y tachada de piltraja humana porque nunca fue atendida por su condición de anciana VIH. del 2009. 
Cervantes, dijo que la figura del anciano ya no goza del respeto de hace muchos años. La falta de respeto al mayor y el culto a la belleza pueden estar arrastrando a muchos en la sociedad a abandonar a sus abuelos o padres de familia.

En este sentido, la inquietud que surge a la hora de abordar un discurso jurídico encomendado para un sector poblacional en situación de vulnerabilidad tiene que ver con los procedimientos para establecer relaciones de poder. Así, la falacia garantista refuerza los procedimientos de lo excluido (la persona vieja) y se jacta de sonreír bajo el escudo protector de una túnica divina que venera el "Imperativo de la Ley" como base del bienestar social.

De esta manera, la palabra no es sólo palabra; orienta, determina, designa y ejecuta relaciones de poder. Por esto, la palabra recibe y determina su existencia y la del sujeto. Así, el discurso del legislador debe ser analizado críticamente, denunciando su lógica emotiva y de forma clara y contundente mostrar que la ley es la presentación de un "debe" como si fuera un "es".

Es decir, lo que se rechaza es el argumento ideológico que se presenta como algo "natural" $y$, por ello, inmodificable (un "debe" presentado como un "es") (Herrera, 2007).

Hace algunos años, se dispuso un apoyo para subsidiar el transporte a 213000 adultos mayores en 3566 rutas públicas de autobuses. De esta forma, los beneficiados podrían utilizar este servicio en forma gratuita si realizan viajes de menos de 25 kilómetros. En trayectorias de entre 25 y 50 kilómetros obtendrán un descuento de 50 por ciento. Y en recorridos de entre 50 y $75 \mathrm{~km}$ tendrán una rebaja del 75 por ciento.

Porque bien lo dice Joaquín Herrera Flores en su Manifiesto Inflexivo: "acá no estamos para pensar desde lo impuesto sino desde la indignación del otro que por las desigualdades ha quedado fuera del contrato social" (Santos, 1998) y así se debe abogar por afirmar esas diferencias defendiendo propuestas y expresando la verdad del sujeto de derechos humanos.

De esta manera, debemos estudiar esas normas jurídicas dictadas por el legislador por- que es por ellas que ese mundo físico y moral se declara respetuoso de ese dogma de voluntad soberana, escrita y hecha ley: manda, prohíbe o permite.

\section{REFERENCIAS BIBLIOGRÁFICAS}

Asamblea Legislativa. Ley Integral del Adulto Mayor de Costa Rica. Ley 7935. San José, Costa Rica. 1999.

Augé, Marc. Los no lugares. Espacios de anonimato. Una antropología de la sobremodernidad. Barcelona, España: Editorial Gedisa, 1994.

Barros, C. Gerontología Básica. Capítulo "El adulto Mayor en la sociedad Chilena". Pontificia Universidad Católica de Chile, miembro Comité Nacional Para el Adulto Mayor Viviendo el envejecer. Pontificia Universidad Católica de Chile. s.f.

Canal 7. Programa Siete Días. La población tica envejece día a día. San José, Costa Rica. 6 De Noviembre de 2006.

Citado en el Informe Anual de Defensoría de los Habitantes. Informes nro. FOE-SO-3/200 y 4/2000 de la División de Fiscalización Operativa y evaluativa en el Área de Servicios Sociales de la Contraloría General de la República. Costa Rica. 1999-2000.

Constitución Politica de Costa Rica (19491999). Edición Conmemorativa de su cincuentenario. San José, Costa Rica.

Defensoría de los Habitantes. Proyecto de Ley Protección del Anciano. Ministerio de Justicia y Gracia. San José, Costa Rica. 1992.

Defensoría de los Habitantes. "Libertad inundada. Algunos casos de 
discriminación en Costa Rica”. Serie los informes de la defensoría. Dirección de promoción y divulgación. San José, Costa Rica: Imprenta Nacional, 2008.

Defensoría de los Derechos Humanos. Proyecto de Ley Protección del Anciano. San José, Costa Rica. Ministerio de Justicia y Gracia. San José, Costa Rica. 1992.

Defensoría de Los Habitantes. Informes nro. FOE-SO-3/200 y 4/2000 de la División de Fiscalización Operativa y evaluativa en el Área de Servicios Sociales de la Contraloría General de la República. Costa Rica. 1999-2000.

Defensoría de Los Habitantes. Informe Anual De Los Habitantes. Costa Rica. San José, Costa Rica. 1997-1998.

Defensoría de Los Habitantes. Informe Anual De Los Habitantes. Costa Rica. San José, Costa Rica. 1998-1999.

Defensoría de Los Habitantes. Informe Anual De Los Habitantes. Costa Rica. San José, Costa Rica. 1999-2000.

Defensoría de Los Habitantes. Informe Anual De Los Habitantes. Costa Rica. San José, Costa Rica. 2000-2001.

Defensoría de Los Habitantes. Informe Anual De Los Habitantes. Costa Rica. San José, Costa Rica. 2001-2002.

Defensoría de Los Habitantes. Informe Anual De Los Habitantes. Costa Rica. San José, Costa Rica. 2002-2003.

Defensoría de Los Habitantes. Informe Anual De Los Habitantes. Costa Rica. San José, Costa Rica. 2004-2005.

Defensoría de Los Habitantes. Informe Anual De Los Habitantes. Costa Rica. San José, Costa Rica. 2006-2007.
Dirección General de Estadística y Censos. Censo Nacional de la Población. San José, Costa Rica. 1984.

Dirección General de Estadística y Censos. Censo Nacional de la Población. San José, Costa Rica. 2000.

División de Población de la CEPAL. Diagnóstico sobre la situación y las políticas de las personas mayores en América Latina y El Caribe. 2003.

Foucault, Michel. "El orden del discurso/L'ordre du discours". Discurso inaugural en el College de France. 1970.

Foucault, Michel. Vigilar y Castigar. Nacimiento de la prisión. México: Editorial Siglo XXI, 2001.

Gobierno de la República. Constitución Política de Costa Rica. Edición Conmemorativa de su cincuentenario 1949-1999. Costa Rica. s.f.

Herrera Flores, Joaquín. Los derechos humanos como productos culturales. Crítica al humanismo abstracto. Madrid: Catarata, 2005.

Hinkelammert, Franz. El asalto al poder mundial y la violencia sagrada del imperio. Departamento Ecuménico de Investigaciones (DEI). San José, Costa Rica. 2003.

Hospital Nacional de Geriatría y Gerontología Dr. Raúl Blanco Cervantes. Basado en la Encuesta de Hogares de 1997. Población adulta mayor en Costa Rica. Principales características socioeconómicas y su impacto en los servicios de salud. San José, Costa Rica. 1998.

Mora, Henry y Hinkelammert, Franz. Hacia una economía para la vida. Preludio a una reconstrucción de la economía. II 
Edición. San José: Editorial Tecnológica de Costa Rica, 2008.

Morin, Edgar. Ciencia con consciencia. Traducción: Ana Sánchez, Barcelona: Edita Anthropos. Editorial del hombre. 1984.

Naciones Unidas. Declaración Universal de los Derechos Humanos 1948. s.f.

Naciones Unidas. Asamblea Mundial sobre el Envejecimiento. Viena, Austria. 1982.

Naciones Unidas. Declaración de Principios y Día Internacional de las Personas Adultas Mayores. 1999.

Naciones Unidas. Plan de Acción Internacional de Viena sobre el envejecimiento. Viena, ONU, Asamblea Mundial Sobre el Envejecimiento. 1982.

Naciones Unidas. World Population. Departamento Asuntos Económicos y Sociales. 1998.

Naciones Unidas. Informe del Estado Nación. Programa de Naciones Unidas para el Desarrollo (PNUD), el Consejo Nacional de Rectores (CONARE) y la Defensoría de los Habitantes. 2000.

Naciones Unidas. Informe Social de la Comisión Económica para América Latina y el Caribe. Panorama Social De América Latina 1999-2000. 2000.

Naciones Unidas. "Perfiles y tendencias". Boletín sobre el envejecimiento Observatorio de personas mayores. Organización Mundial de la Salud. 2001.

Naciones Unidas. Asamblea Mundial sobre el Envejecimiento. Madrid, España. 2002.

Naciones Unidas. Informe de la Segunda Asamblea Mundial sobre el Envejecimiento. Madrid, España. 2002.
Naciones Unidas. Informe del Estado de la Población Mundial. 2002.

Naciones Unidas. Informe de la Conferencia Regional Intergubernamental sobre envejecimiento: Hacia una Estrategia Regional de Implementación para América Latina y el Caribe del Plan de Acción Internacional de Madrid sobre el Envejecimiento. Comisión Económica para América Latina y el Caribe Santiago de Chile. 2003.

Naciones Unidas. Foro de la sociedad civil sobre envejecimiento. Contribución de la Sociedad Civil a la Conferencia Ministerial de la ONU sobre Envejecimiento. s/f.

Naciones Unidas. Declaración de los Derechos del Hombre y del Ciudadano 1789. s/f.

Nunberg, Charlotte. Ageing International XVIII. Las Naciones Unidas adoptan medidas acerca de los principios relacionados con las personas de edad avanzada. 1991.

Organización de Naciones Unidas. Plan de Acción Internacional de Viena sobre el envejecimiento. Asamblea Mundial Sobre el Envejecimiento.Viena, ONU. 1982.

Pérez, A. Derechos Humanos, Estado de Derecho y Constitución. Madrid: Tecnos, 1995.

Periódico La Nación. Sucesos. "Anciano muere debido a incendio causado por candela". $1^{\circ}$ de enero de 2007.

Periódico La Nación. Nacionales. "Más abuso a ancianos". San José, Costa Rica. 16 de junio, 2001.

Periódico Tiempos del Mundo. Chile. Tema Académico. Costa Rica. 14 de febrero, 2002. 
Periódico Tiempos del Mundo. Honduras. Presente Amargo, Futuro Incierto. Costa Rica. 14 de febrero, 2002.

Periódico Tiempos del Mundo. Investigación Especial Sobre El Tema Del Envejecimiento. Costa Rica. 14 de febrero, 2002.

Periódico La Nación. Revista Dominical Tabú entre sábanas. Edición especial. San José, Costa Rica. 15 de julio, 2001.

Periódico La Nación. Más abuso a ancianos. San José, Costa Rica. 16 de junio, 2001.

Periódico La Nación. Editorial. San José, Costa Rica. 23 de diciembre, 2001.
Proño, Leonidas. "Los Derechos Humanos de los Ancianos". Revista los derechos del Pueblo 64. 1991.

Proyecciones de Población (1970-2050). Boletín Demográfico 62. CEPAL/CELADE/ONU. Julio, 1998.

Santos, Boaventura De Sousa. Contrato social y Estado. Editorial. 1999.

Universidad de Costa Rica. Primer estado de situación de la persona adulta mayor en Costa Rica. San José: Editorial UCR, 2008. 